\title{
Validation of SAD PERSONS Scale in Thai Tertiary Context
}

Keerati Pattanaseri, M.D. ${ }^{1}$ Karnthima Nanthasarut, M.D. ${ }^{1}$, Panatchaporn Chantapong, M.D. ${ }^{2}$, Nattha Saisavoey, M.D. ${ }^{1}$

'Department of Psychiatry, Faculty of Medicine Siriraj Hospital, Mahidol University, Bangkok 10700, Thailand. ${ }^{2}$ Krathum Baen Hospital, Krathumbaen, Samuthsakorn 74110, Thailand.

Received 28 May 2020 • Revised 16 August 2020 • Accepted 12 September 2020 • Published online 10 February 2021

\section{Abstract:}

Objective: To examine the validity of the SAD PERSONS scale (SPS) and item analysis in a Thai tertiary setting.

Material and Methods: One hundred and twenty-six inpatients were recruited from a psychiatric ward. Patients were interviewed by a consultant psychiatrist and assessed a variety of suicide risk factors. The SPS was blindly performed by a psychiatric resident. The psychometric properties of SPS was executed by comparing the assessment results.

Results: A cut-off point of SPS was at $2 / 3$ in assessing current high suicide risk with the sensitivity $79.0 \%$ and specificity $35.0 \%$. While the specificity of cut-off point $4 / 5$ was greater than $90.0 \%$, sensitivity was only $27.0 \%$. Parameters that correlated the high risk of suicide were only depression and statement of future suicide intent.

Conclusion: For the purpose of suicide prevention, a sensitivity of screening scale is essential. SPS failed to identify the majority of those requiring high intensity psychological intervention though it shows the high specificity of suicide risk. The instrument should not be applied to screen self-harm patients presenting to general hospitals.

Keywords: psychometric property, suicide risk assessment, SAD PERSONS

Contact: Nattha Saisavoey, M.D.

Department of Psychiatry, Faculty of Medicine Siriraj Hospital, Mahidol University, Bangkok 10700, Thailand.

E-mail: nattha.sai@mahidol.edu

Hosting by Prince of Songkla University. All rights reserved.

This is an open access article under the CC BY-NC-ND license

(http://www.jhsmr.org/index.php/jhsmr/about/editorialPolicies\#openAccessPolicy).

J Health Sci Med Res 2021;39(4):293-302 doi: 10.31584/jhsmr.2021785 www.jhsmr.org 


\section{Introduction}

Suicide is the leading cause of death, by 2016 suicide accounted for $1.4 \%$ of all deaths worldwide. ${ }^{1}$ The suicide situation in Thailand is more serious than in many other regions as Thai suicide rate is 14.1 per 100,000 population which is higher than crude suicide rate of both the SouthEast Asia region (13.2 per 100,000 population) and also the global rate (10.6 per 100,000 population). ${ }^{2}$ The number of years of loss and impact to society increase attention for global prevention strategies, whereas, the majority of patients with suicide attempts who still have a high risk for repeated attempts were not hospitalised. ${ }^{3}$ Risk was not detected during the patients visit. ${ }^{4}$ In an emergency room, suicidal assessment must be done quickly and precisely to maintain patient safety. The risk assessment tools are necessary as it encourages clinicians to classify the patient's severity and make predictions of future behaviour.

Suicide assessment instruments were internationally applied by several healthcare personnel. ${ }^{4-6}$ There are a number of suicidal risk assessments such as the SAD PERSONS scale (SPS) ${ }^{7}$, Beck scale for suicide ideation ${ }^{8}$, Pierce suicide intent scale ${ }^{9}$, ReACT Self-Harm Rule $^{10}$, or Repeated Episodes of Self-Harm score. ${ }^{11}$ To facilitate suicide prevention, a high sensitivity and specificity of suicidal risk assessment scale is essential. Having a reliable assessment scale would be convenient for medical personnel in caring of patients at risk, however, applying only scale is not strongly recommended ${ }^{6,12,13}$ due to their predictive performance. Instead of reliance on only tool, it is suggested that scale should be only a part of suicidal risk assessment, including the current situation, past history, social factors ${ }^{14,15}$ and the instruments should be validated. ${ }^{16}$

The SPS, a 10-item acronym scale is a suicide risk assessment tool. ${ }^{7}$ SPS was debated about its efficacy as it showed area under the curve 0.572 with non-significant statistics in the sample size of 4,019 participants. ${ }^{17}$ In regards to guidelines or efficacy, the SPS has been widely use in Thailand because the mnemonic is simple and works well where both time and manpower are limited. In Thailand, psychiatric house staff are not always rotating through the emergency department ${ }^{18}$ hence the frontline personnel who meet patients with suicide risk have to assess and manage the case. Clinicians also use the scale for clinical judgment to discharge and make appointments at outpatient units or to admit into inpatient care. To date, there has never been a study examining the reliability and validity of SPS in a Thai tertiary care hospital, hence, this study aims to evaluate accuracy of the SPS for identifying individuals at risk for suicide.

\section{Material and Methods}

The study was performed at the psychiatric inpatient unit of Siriraj Hospital, a university hospital in Bangkok, Thailand. The ethical approval was acquired from the Ethics Committee for Research in Humans with a reference number of 319/2558 (EC1) at Siriraj Hospital before participant recruitment. The study population was included from the following criteria; psychiatric inpatients regardless of their psychiatric diagnosis who were admitted during November 2015-October 2016, age above 18 years old and speaking Thai fluently. The exclusion criteria incluced patients who were too frail to participate, comatose, mute, deaf, blind, had cognitive impairment or had severe, aggressive behavior. For individuals with repetition of self-harm, we included only their first time presenting into the study. The reason that we selected inpatients was that there was a number of patients with a high risk suicide, so we could have an appropriate cut-off point for staff on the setting to admit the patient from any units.

A sample size of 167 was calculated, from an estimated admission number of 285 , per year; to give a $5.0 \%$ level significance, with $5.0 \%$ margin of error. All eligible 
patients were invited into our study; however, we could only recruit 126 eligible participants in the one-year time frame.

\section{Baseline patient assessment}

After eligible patients had completed the consent forms, each patient was interviewed comprehensively by a consultant psychiatrist. The interview was done to collect demographic data, review of the current conditions, assess psychiatric conditions, review of previous psychiatric, medical, and developmental history. The assessment classified patients to be high and low suicide risk groups, and the judgment of the consultant psychiatrist was the "gold standard" of suicidal risk classification. After the interview, the physician completed the inpatient medical record form.

\section{Study measure}

SPS is a 10-item scale, with each item corresponding to a risk factor for suicide. Ten criteria are described as following; Gender (male), Age (<19 or $>45$ ), Depression, Previous suicide attempt, Ethanol abuse, Rational thinking loss, Social supports lacking, Organized plan, No spouse and Sickness (chronic debilitating disease). ${ }^{7}$ Each item is scored as 1 if present and 0 if absent on the current presentation. The SPS was assessed by a psychiatric resident, who was trained to use it. It would be performed independently and blinded, which would occur after psychiatrist's assessment. The reason that we chose a psychiatric resident to perform the SPS is it was firstly used for trained medical students to determine suicide risk. Moreover, the SPS generally has been performed by general physicians who graduated from medical schools within three years, and most psychiatric residents in Thailand also graduated from medical schools not more than 5 years. Hence, competency of psychiatric residents in SPS assessment would be compatible with real life situations in Thailand.

\section{Data analysis}

Descriptive analyses were performed using the chisquared or Fisher's exact tests for categorical variables; in case of continuous variables, t- and Mann-Whitney U-tests were used. The associations of continuous variables were performed using Pearson's correlation. Furthermore, predictive performance of SPS was analysed by receiver operating characteristic (ROC) curve. To decide the optimal cut-off points for high suicidal risk, area under the curve $(A \cup C)$ statistics, as well as, sensitivity, specificity, positive predictive value (PPV), negative predictive value (NPV), positive likelihood ratio (+LR) and negative likelihood ratio $(+L R)$ were calculated.

We analysed a group of patients who had stated of future intent to determine whether it has association with suicidal risk. In this study, only the first admission was used from individuals with multiple admissions to maintain the statistical assumption of independence of observation. In the case of missing parameters, only education years data from 4 participants were missed and they were not statistically different from those individuals with available data. Hence, the missing data was removed from the analyses. Backward stepwise logistic regression was conducted to determine the most appropriate model for current suicidal risk. A value of $p<0.050$ was taken as significant. All statistics were analysed using Statistical Package for the Social Sciences Statistics version 18.0.

\section{Results}

\section{Demographic characteristics}

The study population $(n=126)$ was predominantly women (63.5\%) and the mean age was 42.17 years (S.D. $=17.77$, range 18-82). The primary diagnoses leading to admission were affective disorders (50.8\%) and psychotic disorders $(30.2 \%)$. Table 1 shows characteristics of participants in the high and low suicidal risk groups that consisted of 52 people with high risk from in depth interview 
Table 1 Demographic data

\begin{tabular}{|c|c|c|c|c|}
\hline Demographic data & High-risk $(n=52)$ & Low-risk $(n=74)$ & $\mathrm{X}^{2} / \mathrm{t}^{*}$ & p-values \\
\hline Age, mean \pm S.D. & $41.06 \pm 17.63$ & $42.96 \pm 17.94$ & 0.59 & 0.877 \\
\hline \multicolumn{5}{|l|}{ Gender (\%) } \\
\hline Female & $34(65.4)$ & $46(62.2)$ & 0.14 & 0.711 \\
\hline Male & $18(34.6)$ & $28(37.8)$ & & \\
\hline Education (\%) & $50(39.7)$ & $72(57.1)$ & 4.80 & 0.187 \\
\hline No education & $2(4.0)$ & $0(0.0)$ & & \\
\hline Primary education & $5(10.0)$ & $14(19.4)$ & & \\
\hline Secondary education & $14(28.0)$ & $17(23.6)$ & & \\
\hline At least college & $29(58.0)$ & $41(56.9)$ & & \\
\hline Psychiatric diagnosis (\%) & & & 18.54 & $<0.001$ \\
\hline Bipolar disorder & $10(19.2)$ & $22(29.7)$ & & \\
\hline Depressive disorders & $23(44.2)$ & $9(12.2)$ & & \\
\hline Psychotic disorders & $9(17.3)$ & $29(39.2)$ & & \\
\hline Others & $10(19.2)$ & $14(19.4)$ & & \\
\hline More than 1 psychiatric disorders (\%) & $17(32.7)$ & $25(33.8)$ & 0.02 & 0.898 \\
\hline Chronic disease & $20(38.5)$ & $32(43.2)$ & 0.70 & 0.403 \\
\hline Number of chronic sickness & 1.81 & 1.81 & 0.01 & 0.769 \\
\hline SPS scores, mean \pm S.D. & $3.56 \pm 1.24$ & $2.89 \pm 1.12$ & -3.14 & 0.081 \\
\hline
\end{tabular}

Chi-squared test for categorical variables or independent $t$-test for continuous variables were performed to derive the statistics of $\mathrm{X}^{2}$ or $\mathrm{t}$ respectively.

${ }^{\wedge}$ Chronic disease defined as ICD-10 groups of diseases including neoplasms, diseases of the blood and blood-forming organs and certain disorders involving the immune mechanism, endocrine, nutritional and metabolic diseases which is identified from medical records. S.D.=standard deviation, SPS=SAD PERSONS scale

by psychiatrists and 74 people with low risk. In the high risk group, the female gender was predominant (65.4\%), the mean age was slightly less than the low-risk group (41.06 vs. 42.96) and education was lower than the low risk group. No statistically significant difference between the two groups regarding age, gender, education, having of a chronic medical disease, and number of chronic medical disease was observed. Depressive disorders where diagnoses in the high risk (44.2\%) were four times more than in the low risk group (12.2\%), while bipolar disorders (29.7\%) and psychotic disorders (39.2\%) were stand-out in the low risk group. The mean SPS scores of the high risk group was slightly higher than the low risk group $(3.56 \pm 1.24$ and $2.89 \pm 1.12$, respectively) but no statistically significant difference.

\section{Sensitivity analysis}

SPS cut-off points are 3 score categories (low risk 0-4, moderate risk 5-6 and high risk 7-10). However, null patient had SPS score at least 7 in this study, and mean SPS score of the high risk group according to the psychiatrist was only $3.56 \pm 1.24$ which would be classified into low risk group by SPS. The ROC and sensitivity analyses were used to estimate the optimal cut-off point of the SPS as displayed on Table 2 and Figure 1. At cutoff $2 / 3$, the sensitivity for identifying high suicidal risk was $78.9 \%$ and the specificity was $35.1 \%$. While its PPV and NPV were 46.1 and 70.3 , respectively. Though at cutoff $4 / 5$, the PPV and NPV were better than cut-off $2 / 3$, clinicians could have $26.9 \%$ sensitivity and $91.9 \%$ specificity. 
Table 2 Performance of predictive values (\%) of SAD PERSONS 10-score to high-risk suicide

\begin{tabular}{lllllll}
\hline Cut-off points & Sensitivity & Specificity & PPV & NPV & +LR & -LR \\
\hline Score 2/3 & 78.9 & 35.1 & 46.1 & 70.3 & 1.2 & 0.6 \\
Score 3/4 & 46.2 & 74.3 & 55.8 & 66.3 & 1.8 & 0.7 \\
Score 4/5 & 26.9 & 91.9 & 70.0 & 64.2 & 3.3 & 0.8 \\
\hline
\end{tabular}

$P P V=$ positive predictive value, $N P V=$ negative predictive value, $+\mathrm{LR}=$ positive likelihood ratio, $-\mathrm{LR}=$ negative likelihood ratio

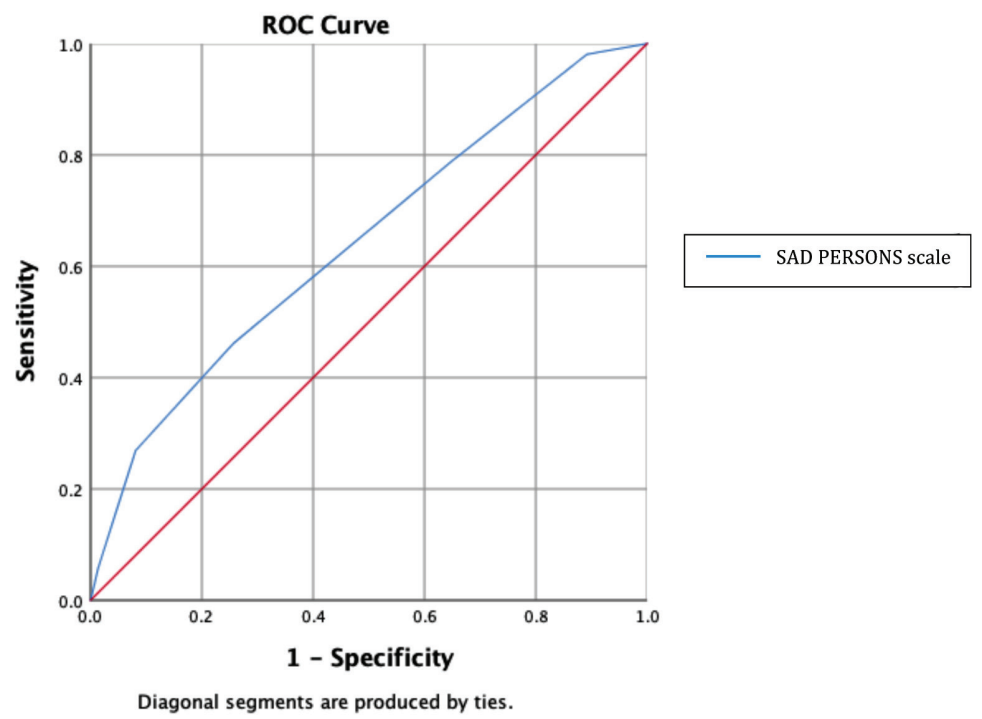

Figure 1 The receiver operating characteristic curves of SAD PERSONS scale, area under the curve to predict highrisk suicide 0.64; 95\% confidence interval 0.55-0.71; p-value $=0.006$

Moreover, figure 1 indicated the AUC for SPS was 0.64 (95\% confidence interval (Cl), 0.55-0.71; p-value=0.006).

Considering the SAD PERSONS mnemonic from univariate analysis (Table 3), depression ( $p$-value<0.001), history of previous suicide gesture $(p=0.010)$, lack of social support ( $p$-value $=0.045$ ), current organized plan for killing oneself ( $p$-value<0.001), and intent to kill oneself in the future ( $p$-value<0.001) were significant factors for current high suicide risk. While two items of SPS showed trends toward negative correlation with current suicidal risk alcohol abuse $(p-$ value $=0.010)$, current rational thinking loss
( $p$-value<0.001). Whereas, these following factors were not significantly associated with high suicidal risk, male sex, age under 19 or over 45 years, no spouse and perception of having chronic sickness. Binary logistic regression analyses were used to determine the association between individual items of SPS and high suicidal risk by a psychiatrist. Table 3 shows the results of the backward stepwise regression analysis of each item of SPS and their relationship to current high suicidal risk. Only depression was correlated with current suicidal risk (adjusted OR=73.21; 95\% Cl, 12.95-413.78). 
Table 3 Association of risk factors with risk assessment and backward stepwise regression model for high suicidal risk

\begin{tabular}{|c|c|c|c|c|c|c|c|}
\hline $\begin{array}{l}\text { Suicide association } \\
\text { factors }\end{array}$ & $\begin{array}{l}\text { High-risk }^{+} \\
(n=52)\end{array}$ & $\begin{array}{l}\text { Low-risk } \\
(n=74)\end{array}$ & $X^{2} / t^{a}$ & $p$-values & $\begin{array}{l}\text { Crude OR } \\
(95 \% \mathrm{Cl})\end{array}$ & $\begin{array}{l}\text { Adjusted Odds } \\
\text { Ratio (95\% CI) } \\
\text { (only SPS) }\end{array}$ & $\begin{array}{l}\text { Adjusted for } \\
\mathrm{D}, \mathrm{P}, \mathrm{O} \text { and SF } \\
(95 \% \mathrm{Cl})\end{array}$ \\
\hline Sex, male & $17(32.7)$ & $28(37.8)$ & 0.35 & 0.553 & $0.80(0.38-1.68)$ & $1.52(0.42-5.46)$ & - \\
\hline $\begin{array}{l}\text { Age }(<19 \text { year or } \\
>45 \text { year })\end{array}$ & $19(36.5)$ & $34(46.0)$ & 1.11 & 0.292 & $0.68(0.33-1.40)$ & $0.80(0.20-3.11)$ & - \\
\hline Depression & $50(96.2)$ & $17(23.0)$ & 65.69 & $<0.001^{b}$ & $\begin{array}{l}83.82 \\
(18.45-380.81)\end{array}$ & $\begin{array}{l}73.21 \\
(12.95-413.78)\end{array}$ & $8.92(1.28-62.33)$ \\
\hline $\begin{array}{l}\text { Previous suicidal } \\
\text { attempt }\end{array}$ & $18(34.6)$ & $11(14.9)$ & 6.72 & 0.010 & $3.03(1.29-7.15)$ & $2.88(0.59-14.04)$ & $2.48(0.48-12.91)$ \\
\hline Ethanol abuse & $3(5.8)$ & $7(9.5)$ & 6.62 & $0.010^{b}$ & $0.24(0.08-0.86)$ & $0.37(0.06-2.21)$ & - \\
\hline Rational thinking loss & $12(23.1)$ & $42(56.8)$ & 14.15 & $<0.001$ & $0.23(0.10-0.51)$ & $0.60(0.17-2.17)$ & - \\
\hline Social support deficit & $6(11.5)$ & $2(2.7)$ & 4.01 & $0.045^{b}$ & $4.70(0.91-24.27)$ & $1.33(0.11-16.50)$ & - \\
\hline Organized plan & $14(28.0)$ & $1(1.4)$ & 19.04 & $<0.001^{\mathrm{b}}$ & $26.90(3.41-212.35)$ & $6.38(0.66-61.94)$ & $1.86(0.19-17.74)$ \\
\hline No spouse & $31(59.6)$ & $42(56.8)$ & 0.10 & 0.749 & $1.13(0.55-2.31)$ & $2.23(0.57-8.73)$ & - \\
\hline Chronic sickness & $14(28.0)$ & $18(24.3)$ & 0.11 & 0.741 & $1.15(0.51-2.58)$ & $0.62(0.15-2.48)$ & - \\
\hline Stated future intent & $47(90.4)$ & $5(6.8)$ & 84.83 & $<0.001$ & $\begin{array}{l}106.53 \\
(30.72-669.50)\end{array}$ & - & $\begin{array}{l}21.36 \\
(4.31-105.77)\end{array}$ \\
\hline
\end{tabular}

${ }^{a}$ Chi-squared test for categorical variables or independent $t$-test for continuous variables were performed to derive the statistics of $\mathrm{X}^{2}$ or $\mathrm{t}$ respectively.

${ }^{b}$ Fisher's exact test was used when the expected values are small

'Chronic disease defined as three ICD-10 groups of diseases including neoplasms, diseases of the blood and blood-forming organs and certain disorders involving the immune mechanism, and endocrine, nutritional and metabolic diseases.

'High-risk group was defined as psychiatrists' assessment.

$\mathrm{D}=$ depression; $\mathrm{O}=$ organized plan, $\mathrm{P}=$ previous suicidal attempt, S.D.=standard deviation, $S F=$ stated future intent, SPS=SAD PERSONS scale

\section{Subgroups analysis}

Groups of patients with chronic disease such as cardiovascular disease, stroke, ischemic heart disease, cancer, diabetes, renal failure, liver failure or cirrhosis, chronic hepatitis B and C virus infection, HIV infection and autoimmune disorder, were analyzed. Table 1 showed that patients report of having chronic diseases, chronic diseases noted from medical records or a subgroup of specified chronic disease did not have significant association with high suicidal risk.

Statement of future intent was another item included in modified SPS but SPS. Fortunately, every patient who was admitted would be assessed with this question. The regression modelling for high risk suicide is presented in Table 3. We selected these four variables, because they have statistical significance at $p$-value $<0.02$, and they are also more significant factors in modified SPS ${ }^{19}$ than others; which give two points for each criteria. Backward stepwise regression selected two items (depression and stated future intent) to be included in the predictive model. Table 3 displays the significant correlation between depression (adjusted OR=8.92; 95\% Cl, 1.28-62.33; p-value<0.001) and intention of future suicide (adjusted OR=21.36; 95\% $\mathrm{Cl}, 4.31-105.77$; $\mathrm{p}$-value<0.001) with high suicidal risk. 


\section{Discussion}

This study demonstrates ability of SPS to assess current suicide risk in Thai tertiary care settings. The SPS scale in this study displays $A U C=0.64 ; p-$ value $=0.006 ; 95 \%$ $\mathrm{Cl}, 0.55-0.74$ that is comparable with the Chinese SPS which has $A \cup C=0.66 ; p-v a l u e=0.020 ; 95 \% \mathrm{Cl}, 0.53-0.79 .^{20}$ There is no consensus in acceptable values of sensitivity and specificity for the predictive performance of suicide assessment scales, in pragmatic view, sensitivity more than $\mathbf{8 0 . 0 \%}$ and specificity more than $50.0 \%$ are acceptable benchmarks. ${ }^{13}$ Using a sample from the tertiary psychiatric center, this study reveals that the SPS does not accurately predict current suicide risk. Since there is no acceptable cut-off value for suicide prediction. For example, at cutoff point 5 clinicians would fail to identify three out of four individuals with high suicide risk that is unacceptable given the seriousness of the consequence. Despite the rather high sensitivity and NPV of the cut-off point 3 , it shows low specificity and PPV. Using this score, clinicians would have an abundancy of false positive cases entering a high intensity treatment program or admissions to psychiatric inpatient care. If that is the case, patients would have overtreated and the medical resources would be extravagantly exhausted. On the other hand, an implication in the Thai tertiary settings is that patients who scored at least 5 would be considered for admission, due to the high risk of suicide; instead of score 7 as the original SPS, with this high specificity of $91.9 \%$. The high risk score, as a priori cut-off points (at least 7), showed sensitivity to be lower than $50.0 \%$ that is worse than a random assessment and with this score more than half of patients with suicide risk would not receive access to an appropriate treatment. Our findings are consistent with other studies that SPS with the high cut-off point had good specificity but poor sensitivity and vice versa. ${ }^{17,20-22}$ The results are also comparable to other suicide risk screening scales, wherein clinicians have to trade-off between sensitivity and specificity, and there is no suicidal risk instrument that can be employed without a comprehensive clinical assessment. ${ }^{15,17}$

We also analysed "stated future intent", a substitution in modified SPS ${ }^{19}$ instead of having chronic sickness. Because statements regarding future suicide intent may imply that they keep thinking that life is not worth living. This scheme combined with access to lethal means, can allow for suicidal behaviours. The study demonstrates a significant association between this parameter with the gold standard diagnosis (adjusted OR=36.44; 95\% Cl, 4.60288.90). The result displays a comparable outcome with several studies ${ }^{23,24}$ that statement regarding future intent to suicide was significantly correlated with a high risk of suicide. Although, we had rather high odds ratio, the $95 \%$ $\mathrm{Cl}$ is rather wide. Hence clinicians can adopt this item to assess the risk yet we could not strongly recommend it. The further investigation to validate modified SPS in a larger sample size than us should be performed to prove whether it would be more applicable in a Thai setting. Another variable that had strong correlation with high suicide risk is depression. These results are similar to several studies. ${ }^{17,19,20}$ It is now understood that depression plays an important role in suicide. This can be explained by cognitive beliefs of depression, which are negative beliefs toward ones self, the world and the future. ${ }^{25}$ People with depression feel alone, helpless and hopeless, which leads into thoughts that life is worthless.

Generally, men have a higher rate of committing suicide than women ${ }^{2}$ and this was also found in the Thai population as committed suicide ratio between men and women was 9.7 to $2.6 .^{24}$ Our result shows this trend but is insignificant (adjusted OR=2.90; 95\% Cl, 0.60-14.18). This might be because we had rather small number of participants and the power of statistics might not be enough. Another reason is that our sample might not reflect the Thai population, in terms of the ratio of women to men as ours was 1.7, however, the Thai women to men ratio is about 
1.0. ${ }^{26}$ Men were more likely to commit suicide than women, whereas, women have more frequently attempted suicide than men. ${ }^{27}$ This might explain why we had a higher number of women in the high risk group than men.

Two items of SPS showed trends toward negative correlation with current suicidal risk; these being alcohol abuse ( $p$-value $=0.010$ ), and current rational thinking loss ( $p$-value<0.001); however, adjusted odd ratio was not significant. This result contrasts with the current knowledge that drug and alcohol misuse has strong association with suicide. ${ }^{28}$ This might be because, we had a small number of alcohol drinking participants (10 of 126), and our study did not collect other substances use data. The negative correlation of rational thinking loss criteria and suicide is similar to Bolton et al. findings. ${ }^{17}$ Rational thinking loss is described as: organic brain syndrome or psychosis ${ }^{19}$, this might relate to impulsive or/and unpredictable behaviors. Thereby, it might increase suicidal risk, if the patient has suicidal ideas, but if they has homicidal ideas attributed to this factor this would have a negative correlation with suicide.

\section{Strengths and limitations}

The study was performed at a tertiary psychiatric center where there is also an academic hospital. Therefore, each case in the study was ensured to be assessed by a psychiatric consultant, so the gold standard was reliable. Due to the limitation of ward occupancy, the condition of each case had to be severe in order to be admitted. Our setting is adequate to validate a screening tool for identifying serious conditions.

There were some limitations to this study. Firstly, our results might not be possible to generalize to the Thai population, since our patients were usually admitted as having a moderate to high clinical severity; therefore, the mean scores reported here were higher than those seen in other units such as emergency or outpatient units. The expected SPS scores should be at least 7, however, our SPS's mean scores are $3.56 \pm 1.24$ in the high risk group and $2.89 \pm 1.12$ in the low risk group. These mean scores reflect that the original SPS scale was not sensitive enough even with the critical group in Thailand. The limitation of having only inpatients in our sample group is that whomever is discharged from emergency or outpatient units were excluded. We also excluded patients who were aggressive and those who had not given their consent. These patients might have different characteristic data from those in the study. Our setting is a tertiary hospital in a medical school, so it seems that our patients might have different characteristics from other settings; such as the severity of both psychiatric and medical conditions. As regards to their medical conditions, up to $50.0 \%$ of our patients have at least one chronic medical illness, which is higher than other settings $\left(15.4^{29}-18.6 \%{ }^{30}\right)$ Hence, our results might not be able to generalize to other settings; except other university hospitals. However, this can be viewed from another angle, in which having a chronic sickness is a risk factor for suicide, our findings should have found this strong association. Likewise, Hockberger et $a{ }^{19}$ removed this item from the modified SPS. This can be interpreted that in a tertiary setting, having a chronic disease might not be associated with high suicide risk. For further investigation, we suggested that all patients who visit the hospital with the issue of suicidal thoughts or attempt should be assessed. Secondly, substance use disorders other than alcohol increases mortality rate from suicide ${ }^{31}$ while we used the original version of SPS which collected only alcohol use information. Hence, we would underestimate the individual's suicide risk. To improve accuracy of suicide risk assessment, data of using both illicit substances including cannabinoid and benzodiazepines should be collected. In addition, modified SPS might be more applicable with contemporary 
context than SPS. Lastly, we included only patients older than 18 years of age so that we would miss the younger age group. Whereas, SPS includes scores of patients who are aged under 19 and more than 45 years old. So we lack the teenage group sample in the study which would affect the results especially relating to age factor.

\section{Conclusion}

The SPS is not recommended to be used as a screening scale of suicide risk presenting at a general hospital. Clinicians should pay attention to clinical assessment that takes account of the individual risk including bio-psycho-social assessment. This skill is a required active training program for all healthcare personnel. In view of specific questions with risk of suicide, both depression and the idea of killing oneself in the future are associated to high risk. So this emphasizes that clinicians who first meet the patient should assess patients with these questions. In regards to the SPS psychometric properties, it might not be sufficient enough to evaluate patients with suicide risk, but it can be used to structure an assessment For developing a further study, not only using a modified SPS instead of SPS to assess current suicide risk but it should also follow participants to predict further suicidal idea or attempts. The modified SPS should be evaluated on its sensitivity and specificity, and also compared with other self-harm instruments. It should be studied in various groups such as non-psychiatric staff to ensure that it can be performed by all healthcare personnel.

\section{Acknowledgement}

We would like to thank Ms Aree Singlamphong and all staff in Siriraj Medical Research Center for their generous support. Crucially, we would like to thank Ms. Naratip Sanguanpanich for helpful guidance and support on handling the data.

\section{Funding sources}

This research project is supported by Siriraj research fund, Faculty of Medicine Siriraj Hospital, Mahidol University.

\section{Conflict of interest}

None

\section{References}

1. World Health Organization. Suicide data [homepage on the Internet] Geneva: WHO; 2020 [cited 2020 Feb 21]. Available from: https://www.who.int/mental_health/prevention/suicide/ suicideprevent/en/

2. World Health Organization. The global health observatory [homepage on the Internet] Geneva: WHO; 2019 [cited 2020 Feb 21]. Available from: https://www.who.int/gho/publications/ world_health_statistics/2019/EN_WHS_2019_Annex2.pdf? ua $=1 \&$ ua $=1$

3. Larkin GL, Smith RP, Beautrais AL. Trends in US emergency department visits for suicide attempts, 1992-2001. Crisis 2008; 29:73-80.

4. Boudreaux ED, Camargo CA Jr, Arias SA, Sullivan AF, Allen $\mathrm{MH}$, Goldstein $\mathrm{AB}$, et al. Improving suicide risk screening and detection in the Emergency Department. Am J Prev Med 2016; 50:445-53.

5. Mitchell AM, Garand L, Dean D, Panzak G, Taylor M. Suicide assessment in hospital emergency departments: implications for patient satisfaction and compliance. Top Emerg Med 2005; 27302-12.

6. National Confidential Inquiry into Suicide and Safety in Mental Health. The assessment of clinical risk in mental health services [homepage on the Internet]. Manchester: The University of Manchester; 2018 [cited 2020 Feb 21]. Available from: https:// sites.manchester.ac.uk/ncish/reports/the-assessmentof-clinical-risk-in-mental-health-services/

7. Patterson WM, Dohn HH, Bird J, Patterson GA. Evaluation of suicidal patients: the SAD PERSONS scale. Psychosomatics 1983;24:343-5.

8. Tyrer $\mathrm{P}$, Henderson $F, M c D e r m o t t ~ U$. Validation of rating scales in psychiatry. Br J Hosp Med 1993;49:434-7. 
9. Pierce DW. The predictive validation of a suicide intent scale: a five-year follow-up. Br J Psychiatry 1981;139:391-6.

10. Steeg S, Kapur N, Webb R, Applegate E, Stewart SL, Hawton $\mathrm{K}$, et al. The development of a population-level clinical screening tool for self-harm repetition and suicide: the ReACT SelfHarm Rule. Psychol Med 2012;42:2383-94.

11. Spittal MJ, Pirkis J, Miller M, Carter G, Studdert DM. The repeated Episodes of Self-Harm (RESH) score: a tool for predicting risk of future episodes of self-harm by hospital patients. J Affect Disord 2014;161:36-42.

12. LeFevre ML, Force USPST. Screening for suicide risk in adolescents, adults, and older adults in primary care: U.S. Preventive Services Task Force recommendation statement. Ann Intern Med 2014;160:719-26.

13. Runeson B, Odeberg J, Pettersson A, Edbom T, Jildevik Adamsson I, Waern M. Instruments for the assessment of suicide risk: a systematic review evaluating the certainty of the evidence. PLoS One 2017;12:e0180292.

14. National Institute for Health and Care Excellence. Self-harm in over 8s: long-term management (Clinical guideline 133). [homepage on the Internet] London: National Institute for Health and Care Excellence; 2011 [cited 2020 Mar]. Available from: https://www.nice.org.uk/guidance/cg133/chapter/ Introduction

15. Quinlivan L, Cooper J, Davies L, Hawton K, Gunnell D, Kapur $N$. Which are the most useful scales for predicting repeat self-harm? A systematic review evaluating risk scales using measures of diagnostic accuracy. BMJ Open 2016;6:e009297.

16. Royal College of Psychiatrists. Self-harm, suicide and risk: a summary [homepage on the internet]. London: Royal College of Psychiatrists; 2010 [cited 2020 Apr]. Available from: https:// www.rcpsych.ac.uk/pdf/PS03-2010x.pdf

17. Bolton JM, Spiwak R, Sareen J. Predicting suicide attempts with the SAD PERSONS scale: a longitudinal analysis. J Clin Psychiatry 2012;73:e735-41.

18. Department of Mental Health, Ministry of Public Health. Annual report 2019. Bangkok: Ministry of Public Health; 2019.

19. Hockberger RS, Rothstein RJ. Assessment of suicide potential by non psychiatrists using the SAD PERSONS score. J Emerg Med 1988;6:99-107.
20. Wu CY, Huang HC, Wu SI, Sun FJ, Huang CR, Liu SI. Validation of the Chinese SAD PERSONS scale to predict repeated self-harm in emergency attendees in Taiwan. BMC Psychiatry $2014 ; 14: 44$.

21. Saunders K, Brand F, Lascelles K, Hawton K. The sad truth about the SAD PERSONS scale: an evaluation of its clinical utility in self-harm patients. Emerg Med J 2014;31:796-8.

22. Quinlivan L, Cooper J, Meehan D, Longson D, Potokar J, Hulme $\mathrm{T}$, et al. Predictive accuracy of risk scales following selfharm: multicentre, prospective cohort study. $\mathrm{Br} \mathrm{J}$ Psychiatry 2017;210:429-36.

23. Chan MK, Bhatti H, Meader N, Stockton S, Evans J, O'Connor $\mathrm{RC}$, et al. Predicting suicide following self-harm: systematic review of risk factors and riskscales. Br J Psychiatry 2016;209: 277-83.

24. Department of Mental Health, Ministry of Public Health. Committed suicide report [homepage on the Internet] Nonthaburi: Ministry of Public Health; 2016 [cited 2020 Apr]. Available from: https://www.dmh.go.th/report/suicide/stat_ sex.asp

25. Beck AT. Thinking and Depression: II. Theory and therapy. Archives of General Psychiatry 1964;10:561-71.

26. The Bureau of Registration Administration, Department of Provincial Administration. Thai population report 2019 [homepage on the internet]. Bangkok: Ministry of Interior; 2019 [cited 2020 Apr 16]. Available from: http://stat.bora.dopa.go.th/stat/ pk/pk_62.pdf

27. Tsirigotis K, Gruszczynski W, Tsirigotis M. Gender differentiation in methods of suicide attempts. Med Sci Monit 2011;17:65-70.

28. Fazel S, Runeson B. Suicide. N Engl J Med 2020;382:26674.

29. Wongsuraprakit S, Santiprasitkul S. Situtation of depression in pre-screened risk groups in Muang district, Chiang Rai province. Thai J Nursing Council 2012;27:91-105.

30. National Statistical Office Thailand. The food consumption behaviour survey. Bangkok: Text \& J; 2017.

31. Schneider B. Substance use disorders and risk for completed suicide. Archives Suicide Res 2009;13:303-16. 